\title{
Resolvin D1 Inhibits Endothelial Permeability and Mitochondrial Damage Following Cardiac Ischemia-Reperfusion in Diabetic Mice
}

\section{Jialiang Zhang}

Sichuan University West China Hospital Department of Cardiology https://orcid.org/0000-0001-77370323

\section{Fangyang Huang}

Sichuan University West China Hospital Department of Cardiology

\section{Li Chen}

Sichuan University West China Hospital Department of Cardiology

\section{Guoyong Li}

Sichuan University West China Hospital Department of Cardiology

\section{Dan Xiao}

Sichuan University West China Hospital Department of Cardiology

Wenhua Lei

Sichuan University West China Hospital Department of Cardiology

Jiahao Zhao

Sichuan University West China Hospital Department of Cardiology

Yanbiao Liao

Sichuan University West China Hospital Department of Cardiology

Changming Li

Sichuan University West China Hospital Department of Cardiology

Mao Chen ( $\square$ hmaochen@vip.sina.com )

Sichuan University West China Hospital Department of Cardiology

\section{Research Article}

Keywords: Myocardial ischemia/reperfusion injury, Resolvin D1, mitochondrial damage, Endothelial permeability, Diabetes.

Posted Date: June 11th, 2021

DOl: https://doi.org/10.21203/rs.3.rs-593100/v1 
License: (c) (i) This work is licensed under a Creative Commons Attribution 4.0 International License. Read Full License 


\section{Abstract \\ Purpose}

Resolvin D1(RvD1), a metabolite derived from docosahexaenoic acid, plays important therapeutic roles in inflammatory diseases. However, the role of RvD1 in diabetic myocardial ischemia/reperfusion (IR) is still unknown.

\section{Methods}

Diabetic mice was established by high-fat diet and streptozotocin, RvD1 was pretreated by intraperitoneal injection for 3 days, followed by myocardial IR. To evaluate the effects of RvD1 on chronic cardiac remodeling, RvD1 was administered for another 2 weeks after IR. The effects of RvD1 following myocardial IR injury were measured, including severity of infarct size, regional inflammation, cardiac function, as well as permeability of cultured endothelial monolayer. Mitochondrial reactive oxygen species (mito-ROS) and mitochondrial membrane potential (MMP) were determined by MitoSOX and JC1.

\section{Results}

RvD1 pretreatment significantly reduced infarct size and the content of Evans blue in injured heart, which was associated with decreased endothelial damage. RvD1 also reduced leukocyte density, gene expression of inflammatory cytokines, cardiomyocytes death, and mitochondrial damage compared to control group. At 2 weeks after myocardial IR, RvD1 treatment partially improved cardiac performance, and reduced cardiac fibrosis in diabetic IR. In vitro, RvD1 attenuated endothelial leakage induced by hypoxia-reoxygenation, $\mathrm{H} 2 \mathrm{O} 2$, and Lipopolysaccharide (LPS), meanwhile, RvD1 also remarkably protected endothelial cells from $\mathrm{H} 2 \mathrm{O} 2$-induced mitochondrial damage as evident from the decreased MMP and increased mito-ROS, which were associated with the preservation of VE-cadherin

\section{Conclusion}

RvD1 protects the heart against diabetic IR-induced injuries by attenuating endothelial permeability and mitochondrial damage. Our study also provide insight into a novel underlying mechanism and a new strategy for treating diabetic IR.

\section{Introduction}

Diabetes mellitus (DM) is a chronic metabolic disorder characterized by insulin resistance and dysfunction of pancreatic beta cells[1]. DM is highly associated with myocardial infarction and heart failure. Obesity, hyperglycemia, and hyperlipidemia are the most common metabolic disorders identified 
in DM and are established cardiovascular risk factors[2, 3]. Diabetic cardiomyopathy is one of the most common complication of DM, which is recognized as a microvascular disease. It has been reported that diabetic cardiomyopathy was associated with higher morbidity and mortality in diabetic patients $[4,5]$. Meanwhile, diabetic heart is more susceptible to myocardial ischemia and reperfusion (IR) and that the cardioprotective effects of ischemic and pharmacological pre-conditioning are compromised in the presence of diabetes[6-8]. Moreover, diabetes induced cardiac dysfunction is associated with endothelial dysfunction that leads to cardiovascular complications [4]. Furthermore, endothelial cells dysfunction represents a crucial step in the development of endothelial cardiomyopathy with diabetes $[9,10]$.Thus, new therapeutic targets prevent the diabetic heart against IR damage are urgently needed.

Resolvin D1 (RvD1), one of the most crucial molecules in specialized pro-resolving mediators (SPMs), which has been proven to play important roles in anti-inflammatory and pro-resolution[11, 12]. Recently, Xia et al have found that RvD1 was remarkably decreased in plasma sample of T2DM patients[13]. Interestingly, another two studies also reported that RvD1 ameliorated streptozotocin-induced diabetes by its anti-inflammatory action, which suggested that RvD1 has potent insulin-sensitizing actions, and might be implicated in the pathogenesis of diabetes[14, 15]. However, it remains unknown whether RvD1 affects vascular permeability in diabetic IR. In the present study, we explored the therapeutic effect of RvD1 on cardiac microvascular damage and explored potential protective mechanisms in diabetic I/R mice.

\section{Methods And Materials}

\section{Myocardial ischemia and reperfusion mice model}

The animal experiment according to the Guide for the Care and Use of Laboratory Animals published by the US National Institutes of Health (NIH Publication No. 85-23, revised 1985). All experimental procedures involving animals were approved by the Ethics Committee of the Animal Care and the Ethics Committee of Sichuan University. For diabetic mice model, 8-10 weeks old male C57BL/6J mice were fed with high fat diet for 4 weeks and then given a single intraperitoneal injection of low-dose STZ $(90 \mathrm{mg} / \mathrm{kg}$, Sigma). One week after STZ injection, mice with 12-hours fasting blood glucose level $\geq 11.1 \mathrm{mmol} / \mathrm{L}$ were considered to have developed diabetes[16,17]. For myocardial ischemia reperfusion model, the left anterior descending (LAD) coronary artery was ligated with a 7-0 suture for 1 hour and then reperfusion for 24 hours. For the sham group, the similar surgical procedure was performed, while the LAD coronary artery was not ligated. RvD1 (Cayman Chemical) was administered to a dose of 50ug/Kg/day by intraperitoneal injection.

\section{Echocardiography}

Echocardiography analysis was performed 14 days after the IR surgery. Mice were anaesthetized with isoflurane, and cardiac function was assessed by an echocardiographic imaging system (GE Vivid 7 equipped with a 12-MHz imaging transducer). Measurements were performed at the midpapillary level from well-aligned M-mode images from the parasternal short axis view, left ventricular ejection fraction (EF\%) and fractional shortening (FS\%), interventricular septum (IVS), left ventricularend diastolic 
dimension (LVID: d), left ventricularend-systolic dimension, (LVID: s), left ventricular-posterior-wall (LVPW), end-diastolic volume (EDV), and end-systolic volume (ESV) were measured.

\section{TTC staining and Evans blue staining}

Following myocardial IR (1 hour/24 hours), 2,3,5-Triphenyltetrazolium chloride (TTC) (Sigma-Aldrich) and Evans blue (Sigma-Aldrich) dye were applied for the measurement of myocardial infract size. Briefly, at the end of reperfusion, $1 \mathrm{~mL} 1 \%$ Evans blue was injected through abdominal aorta after re-ligating the LAD. The heart was then extracted and sectioned once it hardened at $-20^{\circ} \mathrm{C}$, then cut into slices $(2-3$ $\mathrm{mm}$ ). The slices were incubated in the TTC staining solution for 15 minutes at $37^{\circ} \mathrm{C}$. Subsequently, the slices were then fixed in $4 \%$ formaldehyde for 1 hour. Generally, the area stained with Evans blue represented the non-IR myocardium, whereas the unstained area was the I/R myocardium, the red area was at risk, and the white area was infarct size. Images were captured using a camera and the area of the infarcted myocardium in percentage of risk area was analyzed by ImageJ software.

\section{Vascular permeability analysis}

Vascular permeability was evaluated by Evans blue staining as reported[18-20]. In brief, following myocardial IR, at 3 hours before termination of the experiment, Evans blue dye $(20 \mathrm{mg} / \mathrm{kg})$ was injected intravenously, the mice were then euthanized and immediately subjected to aorta perfusion with phosphate buffer saline (PBS). The hearts were then excised, and Evans blue dye was eluted in formamide for 18 hours at $70^{\circ} \mathrm{C}$. The absorbance of Evans blue dye at $620 \mathrm{~nm}$ was measured by Spectra Max 250 spectrophotometer (Molecular Devices, Sunnyvale, CA, USA)

\section{Cell culture}

Human cardiac microvascular endothelial cells (HCMECs) were purchased from Shanghai Huzhen Biotechnology. HCMECs were cultured in DMEM containing $10 \%$ fetal bovine serum and $1 \%$ penicillin/streptomycin in an incubator with $5 \% \mathrm{CO} 2$ at a temperature of $37^{\circ} \mathrm{C}$. The cells were cultured with a final concentration of $30 \mathrm{mM}$ glucose as a high glucose concentration, $5.5 \mathrm{mM}$ glucose condition served as control. Permeability across endothelial cell monolayer was measured in transwell units (with polycarbonate filter, $0.4 \mu \mathrm{m}$ pore; Corning Costar) under following conditions in the presence or absence of RvD1 (100 ng/ml), (1) Hypoxic conditions (5\% CO2 and 94\% N2, 1\% 02,) used fresh DMEM with 1\% FBS to mimic ischemic conditions for 12 hours, reoxygenation at the $5 \% \mathrm{CO} 2$ incubator and with DMEM containing $10 \%$ fetal bovine serum and $1 \%$ penicillin/streptomycin for another 12 hours. H2O2 (400 $\mu \mathrm{M})$ for 12 hours, LPS $(500 \mathrm{ng} / \mathrm{ml})$ for 12 hours. FITC-dextran $(0.5 \mathrm{mg} / \mathrm{ml}$, average molecular mass 40,000 ; Sigma) as a fluorescent indicator was added into the upper-chamber for 2 hours before the end experiments. And $100 \mu \mathrm{l}$ sample was taken from the lower-chamber and the fluorescence of FITC-dextran was measured (485/535 nm, absorption/emission wavelengths) by Spectra Max 250 spectrophotometer (Molecular Devices, Sunnyvale, CA, USA).

\section{Immunofluorescence}


At the final of the cellular experiments, remove the medium, and phosphate buffered saline (PBS) washed for 3 times, then fixed in $4 \%$ paraformaldehyde, permeabilized with 1\% Triton X-100 in PBS, $5 \%$ bovine serum albumin in PBS was used to block cells for $15 \mathrm{~min}$. A primary antibody against VE-cadherin for overnight and a secondary antibody were used for immunostaining 30 minutes, DAPI was used for staining the nucleus for 15 minutes, images were captured by a confocal microscope (N-STORM \& A1, NiKON).

\section{MitosoX staining}

HCMECs were cultured were seeded in 24-well plates with a density of $5 \times 10^{4} / \mathrm{ml}$. The cells were cultured with a final concentration of $30 \mathrm{mM}$ glucose as a high glucose concentration, $5.5 \mathrm{mM}$ glucose condition served as control. RvD1 (100 ng/ml) was pretreaed for 12 hours, followed by $400 \mu \mathrm{M} \mathrm{H} 2 \mathrm{O} 2$ for 4 hours, then cells were washed three times with warm Hank's Balanced Salt Solution (HBSS). MitoSOX-Red (4 $\mu \mathrm{M}$, ThermoFisher Scientific) dye was added to each well, incubated for 30 minutes in the dark at $37^{\circ} \mathrm{C}$, then washed three times with warm HBSS. Finally, fluorescence intensity was determined by Celigo imaging cytometer, images were captured by a confocal microscope (N-STORM \& A1, NiKON).

\section{Mitochondrial transmembrane potential (MMP) assay}

Mitochondrial membrane potential detection was used JC-1 (MedChemExpress Cat. No.: HY-K0601). According to the manufacturer's instructions, JC-1 (2 $\mu \mathrm{M})$ was added to per well, and incubated at $37^{\circ} \mathrm{C}$ for 20 minutes, followed by centrifuged for 3 minutes at 400g, washed cells twice with PBS, and finally added $500 \mu \mathrm{L}$ PBS to suspend cells. JC-1 fluorescence was measured with a flow cytometer.

\section{Western blotting}

HCMECs or heart tissue were lysed using Lysis Buffer (Beyotime) with protease inhibitors (CST). Protein lysates were separated by SDS-PAGE, then transferred to a PVDF membrane, and blotted with antibodies against the target proteins [VE-cadherin (BIOSS), 1:1000 dilution; GAPDH (Affinity Biosciencesy), 1:1000 dilution], followed by a goat Horseradish Peroxidase (HRP)-conjugated antibody (1:10,000 dilution), bands were detected by an ECL system.

\section{Immunostaining analysis}

Immunostaining for heart sections, hearts were excised from mice after euthanasia, fixed in $4 \%$ paraformaldehyde overnight, embedded in paraffin, and sectioned. The sections were then deparaffinized, rehydrated, retrieved for antigens, and subjected to immunohistochemical analysis using against CD45 (Abcam), CD68(Abcam), followed by staining with a secondary antibody (goat anti-rabbit antibody). Images were captured by ZEISS IX83.For Masson staining, heart sections were deparaffinized, stained with Masson's composite staining solution and a bright-green staining solution and dehydrated in absolute ethanol. Acetic acid was used for washing. Finally, the sections were subjected to transparency in xylene and then mounted with neutral gum for analysis. 


\section{Quantitative real-time reverse transcription-polymerase chain reaction(qRT-PCR)}

The total RNA was extracted from tissues or cell lysates using Trizol (Invitrogen), after extracting using chloroform and precipitating with isopropanol, the RNA was washed twice using $75 \%$ ethanol, and the concentration of RNA was measurement after dissolved in RNase-free water. Subsequently the RNA was reversely transcribed by the PrimeScript RT Reagent Kit (Takara). qRT-PCR assay was performed on the CFX96TM Real-time PCR Detection System (BioRad) using EvaGreen Supermix Kit (Bio-Rad). Relative mRNA expression was normalized to GAPDH. The qPCR primer sequences were as following Table 1:

Table 1. Primers for quantitative real-time PCR.

\begin{tabular}{|lll|}
\hline Genes & Forward primer sequence $\left(5^{\prime}\right.$-3') $^{\prime}$ & Reverse primer sequence $\left(5^{\prime}\right.$-3 $\left.^{\prime}\right)$ \\
\hline mGAPDH & ATGGTGAAGGTCGGTGTGAACG & GGTCTCGCTCCTGGAAGATGGT \\
\hline m-IL-1 $\beta$ & CTTCAGGCAGGCAGTATC & CAGCAGGTTATCATCATCATC \\
m-MCP-1 & CCACTCACCTGCTGCTACTCA & TGGTGATCCTCTTGTAGCTCTCC \\
m-IL-6 & TGTGCAATGGCAATTCTGAT & GGTACTCCAGAAGACCAGAGGA \\
\hline hGAPDH & TGACCACAGTCCATGCCATCAC & ACGCCTGCTTCACCACCTTCT \\
\hline h-IL-1 $\beta$ & AAG CTG ATG GCC CTA AAC AG & AGG TGC ATC GTG CAC ATA AG \\
\hline h-IL-6 & ATGAACTCCTTCTCCACAAGC & AAGAGCCCTCAGGCTGGACTG \\
\hline h-MCP-1 & CATAGCAGCCACCTTCATTC & TCTGCACTGAGATCTTCCTATTGG \\
\hline
\end{tabular}

\section{Transmission electron microscope (TEM)}

Heart tissues were harvested and quickly fixed in $2.5 \%$ glutaraldehyde for 1 hours, exposed to $1 \%$ osmium tetroxide, then sectioned to $60 \mathrm{~nm}$ and mounted on Cu-grids contrasted with uranyl acetate and lead citrate, and analyzed using a JEM-1400 electron microscope.

\section{Statistical analysis}

All experimental data were presented as means \pm SD. The difference between 2 groups of variables was compared by the 2-tailed, unpaired T-test. For data analysis of more than 2 groups, one-way ANOVA test was used. A value of $P<0.05$ was considered to be statistically significant.

\section{Results}

RvD1 pretreatment protects against vascular hyperpermeability after myocardial IR injury in diabetic mice 
After successful established diabetic mice model. DM+IR+ RvD1 group were pretreated RvD1 for 3 days. After myocardial ischemia reperfusion operation. An assay with Evans blue dye and TTC staining was performed to determine myocardial infarct size after IR, TTC staining showed that pretreatment with RvD1 significantly reduced the infarct size by $12 \%$ compared with I/R group in diabetic mice. As shown in (Fig. 2C, 2D). Vascular leakage was measured by Evn's blue extravasation after myocardial IR, as the results presentation, Evan's blue increased after I/R injury in diabetic mice, however, the effect was inhibited by RvD1treatment (Fig. 2E, 2F). These data suggest that RvD1 provided myocardial protection against I/R injury by inhibiting vascular permeability in diabetes.

\section{RvD1 pretreatment inhibited inflammatory responses in diabetic I/R injury}

The TEM images of endothelial barrier demonstrated that endothelial cells from the diabetic I/R heart appeared swollen with increased lysosomal-like inclusions and the integrity of endothelial barrier was also damaged, while pretreated with RvD1 could alleviated endothelial barrier damage (Fig. 4A, 4B). The inflammation in response to diabetic IR was evaluated by immunohistochemical detection of the density of inflammatory cells (CD45 and CD68) in hearts (Fig. 3A, 3B), gene expression of inflammatory cytokines (IL-1 $\beta$, IL-6 and MCP-1) in diabetic IR hearts (Fig. 3C). AS the results showed that there was a robust increase in the density of leukocytes CD45 and macrophages CD68 in vehicle-treated group. Above all, RvD1 pretreatment therapy reduced the density of inflammatory cells, and also significantly downregulated expression of IL-1 $\beta$, IL- 6 and MCP-1 versus vehicle treated group.

\section{RvD1 pretreatment improved cardiac performance and alleviated chronic cardiac remodeling}

To study the influence of RvD1 treatment on chronic cardiac remodeling, RvD1 was administered via intraperitoneal injection ( $50 \mu \mathrm{g} / \mathrm{kg} /$ day) for another 2 weeks after l/R. Echocardiography was performed at 2 weeks after IR. Mice with IR exhibited significantly decline in EF and FS (Fig. 5A). Comparison with untreated mice, RvD1 treated mice had a significantly improvement of EF and FS. To assess effect of RvD1 on cardiac fibrosis, Masson staining was performed to evaluate cardiac remodeling after IR injury (Fig. 5B, 5C), the results showed that fibrosis was increased after IR injury, while RvD1 treatment alleviated cardiac fibrosis at 2 weeks after IR in diabetes.

\section{RvD1 improved permeability of endothelial cells stimulated with different pathological conditions}

To assess the effect of RvD1 on endothelial permeability, we used transwell device to grow HCMECs into a monolayer in the condition of high glucose. Cells were subjected to 12 hours hypoxia following by reoxygenation of 12 hours $(\mathrm{H} / \mathrm{R})$. Meanwhile various stimuli, including $\mathrm{H} 2 \mathrm{O} 2$ or LPS were added to the culture medium with or without the pretreatment of RvD1 for another 2 hours. The dextran-FITC of lower chamber was etected to determine endothelial permeability. Fluorescence intensity of FITC in lower chamber was significantly higher after H/R (Fig. 6B), H2O2 (Fig. 6C) and LPS (Fig. 6D) stimulation, but it was attenuated with RvD1 treatment. Q-PCR analysis showed that RvD1 treatment significantly suppresses H/R-induced expression of inflammatory genes MCP-1, IL-6, and IL-1 $\beta$ mRNA expression in high glucose condition (Fig. 6E). Taken together, these findings suggested that RvD1 attenuate HR, H2O2 
and LPS-induced endothelial permeability and decrease H/R-induced inflammation in high glucose condition.

\section{RvD1-mediated microvascular protection was regulated by mitochondrial damage}

Elevated ROS levels have been shown to have important roles in regulating endothelial barrier. First, MitoSOX probe was used to examine the cellular level of ROS cultured with high glucose DMEM. As the results presented that $\mathrm{H} 2 \mathrm{O} 2$ significantly increased mito-ROS, while RvD1 treatment decreased mito-ROS level in high glucose condition (Fig. 7A, 7B). Second, as mitochondrial transmembrane potential (MMP) plays a key role in mitochondrial homeostasis through selective elimination of dysfunctional mitochondria[21], JC-1 was used to detect the MMP. The flowcytometry results revealed RvD1 pretreatment significantly mitigated mitochondrial membrane potential drop induced by $\mathrm{H} 2 \mathrm{O} 2$ in high glucose condition (Fig. 7C).

\section{RvD1 inhibited endothelial permeability with preservation of VE-cadherin}

As VE-cadherin-mediated cell-cell adhesion organizes the endothelial junctions and maintains the barrier function, which is central in permeability changes. Thus, we investigated the effect of RvD1 on the expression levels of VE-cadherin. We used immunofluorescence and western blots examined the cellular level of VE-cadherin cultured with high glucose DMEM after H/R. Immunofluorescence and western blot analysis showed that RvD1 treatment significantly increased the level of VE-cadherin after H/R (Fig. 8A, $8 B$ ). Meanwhile, VE-cadherin was also detected in the diabetic heart tissue after $I / R$, the results also revealed that VE-cadherin was decreased in diabetic heart tissue after IR, which was reversed by RvD1 pretreatment (Fig. 8C).

Therefore, these evidences suggested that the effect of RvD1 on the endothelial permeability may be mediated by regulating the expression of VE-cadherin.

\section{Discussion}

Numerous studies report that high glucose environments aggravate myocardial vulnerability to ischemia/reperfusion injury[22-24]. For the first time, we provide evidence that RvD1, an important member of endogenous specialized pro-resolving mediators, provides cardio-protection from myocardial ischemia reperfusion injury in diabetic mice by restricting endothelial permeability. First, RvD1 pretreatment significantly decreased myocardial content of Evans blue and reduced infarct size in diabetic IR mice. Second, RvD1 pretreatment of was associated with suppression of regional inflammatory responses, alleviation of cardiomyocytes death, consequently treated with RvD1 improved cardiac performance and restricted cardiac remodelling. Finally, RvD1 pretreatment protected endothelial cell monolayer against endothelial leakage induced by hypoxia/re-oxygenation, $\mathrm{H} 2 \mathrm{O} 2$ or Lipopolysaccharide, effects that are associated with preservation of VE-cadherin. 
Resolvin D1, derived from the omega-3 fatty acids docosahexaenoic acid, reveals remarkable potentiality in resolving inflammation-related diseases such as abdominal aortic aneurysm formation, sepsis [12, 25-27]. Recently, Xia et al have found that RvD1 was remarkably decreased in Type 2 diabetes mellitus (T2DM) patients' plasma samples versus healthy controls, indicating that RvD1 may play important role in the pathogenesis of T2DM[13]. While, in our present study, RVD1 treatment alleviated the endothelial permeability in diabetic I/R mice without influencing the fasting glucose.

Several studies reported that RvD1 prevented LPS-induced polymorphonuclear leukocytes recruitment to endothelial cells, and attenuated endothelial barrier permeability[28-30]. Moreover, stabilization of the vasculature by RvD1 maybe another potential molecular mechanism underlying the protection of RvD1 on diabetic IR heart. It is well-known that VE-cadherin is a component of endothelial cell-to-cell adherens junctions, and is an important determinant of the barrier function of the vascular endothelium[31]. Previous studies reported that preservation of VE-cadherin confer cardio-protection in myocardial I/R[18, 19]. Rima Chattopadhyay et al have also found that RvD1 attenuated LPS-induced adherens junction disassembly and endothelial barrier permeability by arresting tyrosine phosphorylation of a-catenin and VE-cadherin[28]. Our results also reported that RvD1 pretreatment inhibited endothelial monolayer leakage via regulating the expression of VE-cadherin.

On the other hand, a burst of reactive oxygen species sourced from mitochondria at the beginning of the reperfusion, aggravate myocardial injury through a number of different mechanisms[32, 33]. Therefore, mitochondrial damage determines myocardial damage during IR and subsequent remodeling[34-36]. RvD1 has already been proven to reduce reactive oxygen species levels, improve mitochondrial morphology and function, promote mitochondrial DNA repair in primary retinal cells with diabetic retinopathy[37]. In diabetes, high blood glucose and free fatty acids induce ECs mitochondrial fission, with high levels of reactive oxygen species (ROS), leading to blunted cell growth and altered cell-adhesion molecule expression on the cell surface[38]. In our study, the results were consistent with those studies that RvD1 pretreatment not only reduced cardiomyocytes apoptosis, but also subsequently treatment with RvD1 also attenuated pathological cardiac remodeling after IR in diabetic mice. At the same time, the results indicated that RvD1 deceased mito-ROS generation stimulated with $\mathrm{H} 2 \mathrm{O} 2$ in high glucose condition. Mitochondrial transmembrane potential (MMP) is also an important parameter of the mitochondrial function which is considered as an indicator of cell health[21]. During apoptosis, MMP decreases is associated with the opening of the mitochondrial permeability pores and loss of the electrochemical gradient. In the present study, we found that RvD1 prevented H2O2-induced MMP reduction. While, further studies are needed to better understand the underlying mechanisms of RvD1 in regulating mitochondrial function in cardiovascular diseases.

In summary, RvD1 attenuated myocardial microvascular damage following diabetic cardiac IR injury, which presented with reduction of infarct size, suppression of inflammatory response, and decreased cardiomyocyte apoptosis. The underlying molecular mechanism is partially through preservation of VEcadherin and modulating mitochondria function. Therefore, RvD1 treatment may be a promising therapeutic drug to protect diabetic heart against ischemia and reperfusion.

Page 10/22 


\section{Declarations}

\section{Conflict of Interest}

The authors declare that they have no conflict of interest.

\section{Ethical Approval}

All applicable international, national, and/or institutional guidelines for the care and use of animals were followed.

\section{Informed Consent}

Informed consent was obtained from all individual participants included in the study.

Author Contributions: MC, CL, FY, and JZ initiated the project and were responsible for the design of the protocol. LY did the literature review. JZ and FY analyzed the data.LC, GL, and WL finished the animal experiments. JZ, FY, and LC composed the initial draft of the manuscript. JZ, WH, DX, GY, LC, JZ (Jiahao Zhao), and $Y L$ were responsible for critical revision of the manuscript and provided important intellectual content. All authors approved the final version of the manuscript submitted for publication.

Funding: This study was supported by The National Natural Science Foundation of China (Grant Nos. 81900258, 8197020724), The Sichuan Science and Technology Program (Grant Nos. 2020YFS0246.), the China Postdoctoral Science Foundation Grant (Grant Nos: 2019M663521), the Post-Doctor Research Project, Sichuan University (2020SCU12029), the Post-Doctor Research Project, West China Hospital, Sichuan University (Grant Nos: 2019HXBH016).

Availability of data and material: The data used to support the findings of this study are transparency and could be available from the submitting author (zj1094@126.com) upon request.

\section{References}

1. Nolan CJ, Damm P, Prentki M. Type 2 diabetes across generations: from pathophysiology to prevention and management. Lancet. 2011;378:169-81. 10.1016/S0140-6736(11)60614-4.

2. Ritchie RH, Abel ED. Basic Mechanisms of Diabetic Heart Disease. Circ Res. 2020;126:1501-25. 10.1161/CIRCRESAHA.120.315913.

3. Russo I, Penna C, Musso T, Popara J, Alloatti G, Cavalot F, Pagliaro P. Platelets, diabetes and myocardial ischemia/reperfusion injury. Cardiovasc Diabetol. 2017;16:71. 10.1186/s12933-0170550-6.

4. Horton WB, Barrett EJ. Microvascular Dysfunction in Diabetes Mellitus and Cardiometabolic Disease. Endocr Rev. 2021;42:29-55. 10.1210/endrev/bnaa025. 
5. Ugusman A, Kumar J, Aminuddin A. Endothelial function and dysfunction: Impact of sodium-glucose cotransporter 2 inhibitors. Pharmacol Ther. 2021;224:107832. 10.1016/j.pharmthera.2021.107832.

6. Donahoe SM, Stewart GC, McCabe CH, Mohanavelu S, Murphy SA, Cannon CP, Antman EM. Diabetes and mortality following acute coronary syndromes. JAMA. 2007;298:765-75. 10.1001/jama.298.7.765.

7. Dauriz M, Morici N, Gonzini L, Lucci D, Di Chiara A, Boccanelli A, Olivari Z, Casella G, De Luca L, Temporelli P, De Servi S, Bonora E, Savonitto S. Fifteen-Year Trends of Cardiogenic Shock and Mortality in Patients with Diabetes and Acute Coronary Syndromes. Am J Med. 2020;133:331-9 e332. 10.1016/j.amjmed.2019.07.044.

8. Penna C, Andreadou I, Aragno M, Beauloye C, Bertrand L, Lazou A, Falcao-Pires I, Bell R, Zuurbier CJ, Pagliaro P, Hausenloy DJ. Effect of hyperglycaemia and diabetes on acute myocardial ischaemiareperfusion injury and cardioprotection by ischaemic conditioning protocols. $\mathrm{Br} \mathrm{J}$ Pharmacol. 2020;177:5312-35. 10.1111/bph.14993.

9. Paneni F, Beckman JA, Creager MA, Cosentino F. Diabetes and vascular disease: pathophysiology, clinical consequences, and medical therapy: part I. Eur Heart J. 2013;34:2436-43. 10.1093/eurheartj/eht149.

10. Knapp M, Tu X, Wu R. Vascular endothelial dysfunction, a major mediator in diabetic cardiomyopathy. Acta Pharmacol Sin. 2019;40:1-8. 10.1038/s41401-018-0042-6.

11. Serhan CN. Pro-resolving lipid mediators are leads for resolution physiology. Nature. 2014;510:92101. 10.1038/nature13479.

12. Serhan $\mathrm{CN}$, Chiang N, Van Dyke TE. Resolving inflammation: dual anti-inflammatory and proresolution lipid mediators. Nat Rev Immunol. 2008;8:349-61. 10.1038/nri2294.

13. Xia F, He C, Ren M, Xu FG, Wan JB. Quantitative profiling of eicosanoids derived from n-6 and n-3 polyunsaturated fatty acids by twin derivatization strategy combined with LC-MS/MS in patients with type 2 diabetes mellitus. Anal Chim Acta. 2020;1120:24-35. 10.1016/j.aca.2020.04.064.

14. Bathina S, Gundala NKV, Rhenghachar P, Polavarapu S, Hari AD, Sadananda M, Das UN. Resolvin D1 Ameliorates Nicotinamide-streptozotocin-induced Type 2 Diabetes Mellitus by its Anti-inflammatory Action and Modulating PI3K/Akt/mTOR Pathway in the Brain. Arch Med Res. 2020;51:492-503. 10.1016/j.arcmed.2020.05.002.

15. Bathina S, Das UN. Resolvin D1 Decreases Severity of Streptozotocin-Induced Type 1 Diabetes Mellitus by Enhancing BDNF Levels, Reducing Oxidative Stress, and Suppressing Inflammation, Int J Mol Sci 22 (2021). 10.3390/ijms22041516.

16. Ding M, Dong Q, Liu Z, Liu Z, Qu Y, Li X, Huo C, Jia X, Fu F, Wang X. Inhibition of dynamin-related protein 1 protects against myocardial ischemia-reperfusion injury in diabetic mice. Cardiovasc Diabetol. 2017;16:19. 10.1186/s12933-017-0501-2.

17. Ding M, Hu L, Yang H, Gao C, Zeng K, Yu M, Feng J, Qiu J, Liu C, Fu F, Li Y. Reduction of SIRT1 blunts the protective effects of ischemic post-conditioning in diabetic mice by impairing the Akt signaling pathway. Biochim Biophys Acta Mol Basis Dis. 2019;1865:1677-89. 10.1016/j.bbadis.2019.04.005. 
18. Li Y, Yao Y, Li J, Chen Q, Zhang L, Wang QK. Losartan protects against myocardial ischemia and reperfusion injury via vascular integrity preservation. FASEB J. 2019;33:8555-64. 10.1096/fj.201900060R.

19. Gao XM, Su Y, Moore S, Han LP, Kiriazis H, Lu Q, Zhao WB, Ruze A, Fang BB, Duan MJ, Du XJ. Relaxin mitigates microvascular damage and inflammation following cardiac ischemia-reperfusion. Basic Res Cardiol. 2019;114:30. 10.1007/s00395-019-0739-9.

20. Radu M, Chernoff J. An in vivo assay to test blood vessel permeability, J Vis Exp (2013) e50062. $10.3791 / 50062$.

21. Sivandzade F, Bhalerao A, Cucullo L. Analysis of the Mitochondrial Membrane Potential Using the Cationic JC-1 Dye as a Sensitive Fluorescent Probe, Bio Protoc 9 (2019). 10.21769/BioProtoc.3128.

22. Gan L, Xie D, Liu J, Bond Lau W, Christopher TA, Lopez B, Zhang L, Gao E, Koch W, Ma XL, Wang Y. Small Extracellular Microvesicles Mediated Pathological Communications Between Dysfunctional Adipocytes and Cardiomyocytes as a Novel Mechanism Exacerbating Ischemia/Reperfusion Injury in Diabetic Mice. Circulation. 2020;141:968-83. 10.1161/CIRCULATIONAHA.119.042640.

23. Lejay A, Fang F, John R, Van JA, Barr M, Thaveau F, Chakfe N, Geny B, Scholey JW. Ischemia reperfusion injury, ischemic conditioning and diabetes mellitus. J Mol Cell Cardiol. 2016;91:11-22. 10.1016/j.yjmcc.2015.12.020.

24. Prasad A, Stone GW, Stuckey TD, Costantini CO, Zimetbaum PJ, McLaughlin M, Mehran R, Garcia E, Tcheng JE, Cox DA, Grines CL, Lansky AJ, Gersh BJ. Impact of diabetes mellitus on myocardial perfusion after primary angioplasty in patients with acute myocardial infarction. J Am Coll Cardiol. 2005;45:508-14. 10.1016/j.jacc.2004.10.054.

25. Wang M, Liu M, Zhang J, Liu J, Ye J, Xu Y, Wang Z, Ye D, Zhao M, Wan J. Resolvin D1 protects against sepsis-induced cardiac injury in mice. Biofactors. 2020;46:766-76. 10.1002/biof.1668.

26. Pope NH, Salmon M, Davis JP, Chatterjee A, Su G, Conte MS, Ailawadi G, Upchurch GR Jr. D-series resolvins inhibit murine abdominal aortic aneurysm formation and increase $\mathrm{M} 2$ macrophage polarization. FASEB J. 2016;30:4192-201. 10.1096/fj.201600144RR.

27. Gerlach BD, Marinello M, Heinz J, Rymut N, Sansbury BE, Riley CO, Sadhu S, Hosseini Z, Kojima Y, Tang DD, Leeper NJ, Spite M, Barroso M, Rayner KJ, Fredman G. Resolvin D1 promotes the targeting and clearance of necroptotic cells. Cell Death Differ. 2020;27:525-39. 10.1038/s41418-019-0370-1.

28. Chattopadhyay R, Raghavan S, Rao GN. Resolvin D1 via prevention of ROS-mediated SHP2 inactivation protects endothelial adherens junction integrity and barrier function. Redox Biol. 2017;12:438-55. 10.1016/j.redox.2017.02.023.

29. Chattopadhyay R, Mani AM, Singh NK, Rao GN. Resolvin D1 blocks H2O2-mediated inhibitory crosstalk between SHP2 and PP2A and suppresses endothelial-monocyte interactions. Free Radic Biol Med. 2018;117:119-31. 10.1016/j.freeradbiomed.2018.01.034.

30. Miyahara T, Runge S, Chatterjee A, Chen M, Mottola G, Fitzgerald JM, Serhan CN, Conte MS. D-series resolvin attenuates vascular smooth muscle cell activation and neointimal hyperplasia following vascular injury. FASEB J. 2013;27:2220-32. 10.1096/fj.12-225615. 
31. Giannotta M, Trani M, Dejana E. VE-cadherin and endothelial adherens junctions: active guardians of vascular integrity. Dev Cell. 2013;26:441-54. 10.1016/j.devcel.2013.08.020.

32. Tan Y, Mui D, Toan S, Zhu P, Li R, Zhou H. SERCA Overexpression Improves Mitochondrial Quality Control and Attenuates Cardiac Microvascular Ischemia-Reperfusion Injury. Mol Ther Nucleic Acids. 2020;22:696-707. 10.1016/j.omtn.2020.09.013.

33. Wang J, Zhou H. Mitochondrial quality control mechanisms as molecular targets in cardiac ischemia-reperfusion injury. Acta Pharm Sin B. 2020;10:1866-79. 10.1016/j.apsb.2020.03.004.

34. Bugger $\mathrm{H}$, Pfeil K. Mitochondrial ROS in myocardial ischemia reperfusion and remodeling. Biochim Biophys Acta Mol Basis Dis. 2020;1866:165768. 10.1016/j.bbadis.2020.165768.

35. Vasquez-Trincado C, Garcia-Carvajal I, Pennanen C, Parra V, Hill JA, Rothermel BA, Lavandero S. Mitochondrial dynamics, mitophagy and cardiovascular disease. J Physiol. 2016;594:509-25. 10.1113/JP271301.

36. Wang J, Toan S, Zhou H. New insights into the role of mitochondria in cardiac microvascular ischemia/reperfusion injury. Angiogenesis. 2020;23:299-314. 10.1007/s10456-020-09720-2.

37. Trotta MC, Pieretti G, Petrillo F, Alessio N, Hermenean A, Maisto R, D'Amico M. Resolvin D1 reduces mitochondrial damage to photoreceptors of primary retinal cells exposed to high glucose. J Cell Physiol. 2020;235:4256-67. 10.1002/jcp.29303.

38. Zhu W, Yuan Y, Liao G, Li L, Liu J, Chen Y, Zhang J, Cheng J, Lu Y. Mesenchymal stem cells ameliorate hyperglycemia-induced endothelial injury through modulation of mitophagy. Cell Death Dis. 2018;9:837. 10.1038/s41419-018-0861-x.

\section{Figures}

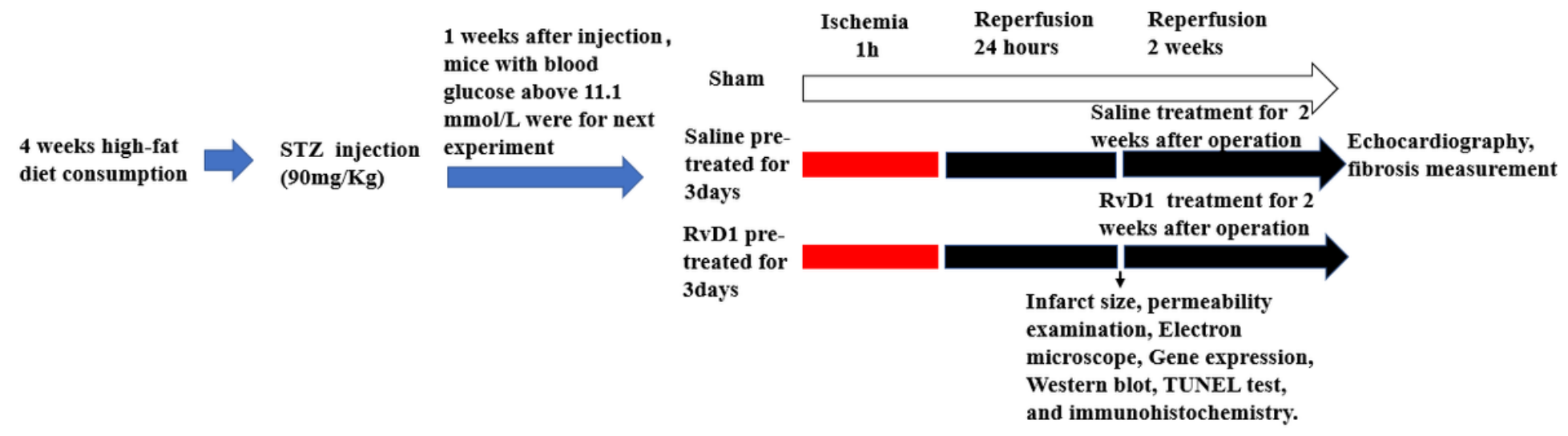

Figure 1

Study design and experimental protocol. 

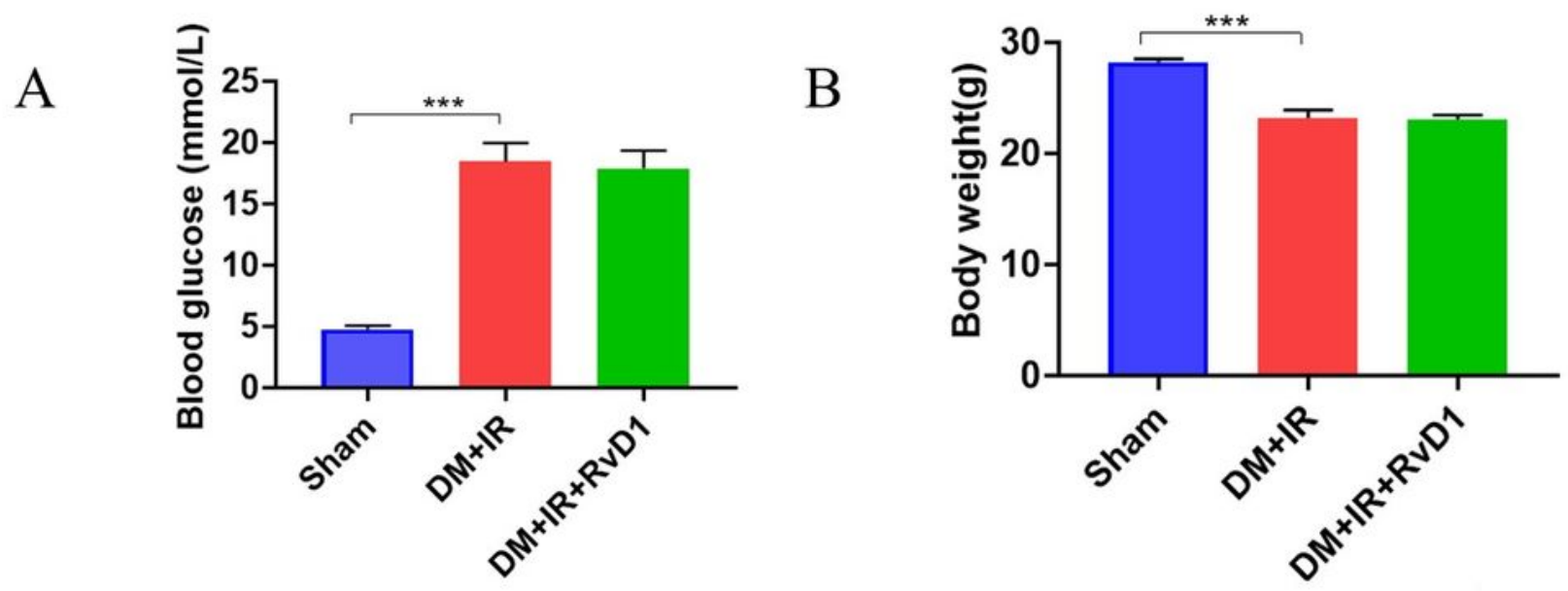

$\mathrm{C}$

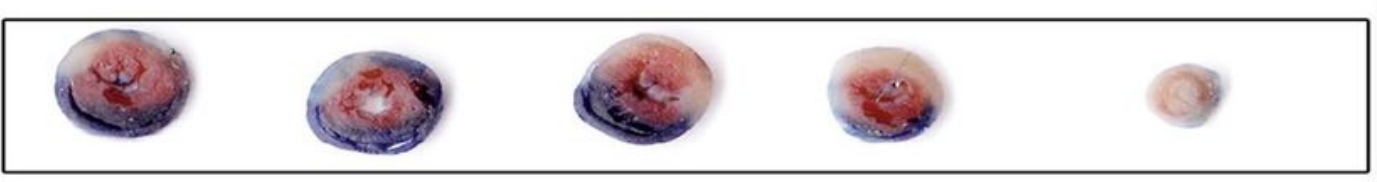

$\mathbf{D M}+\mathbf{I} / \mathbf{R}$

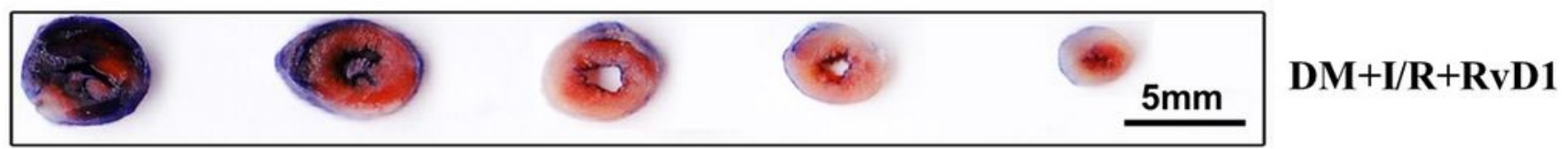

$\mathrm{D}$

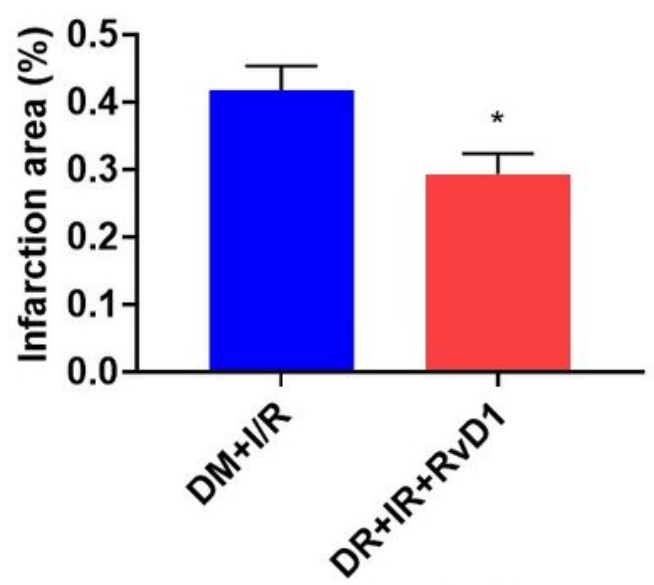

$\mathrm{E}$

$$
\mathbf{D M}+\mathbf{I} / \mathbf{R}
$$

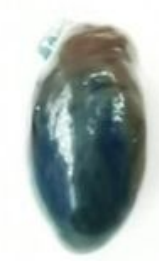

DM+I/R+RvD1

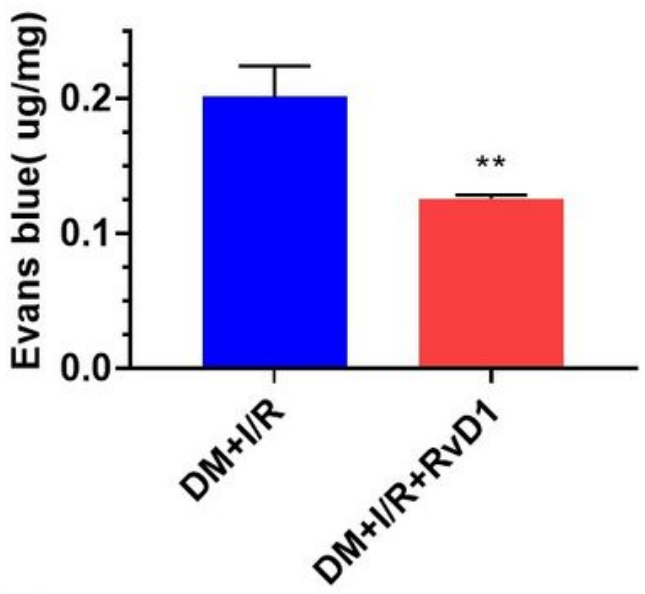

$\mathrm{F}$

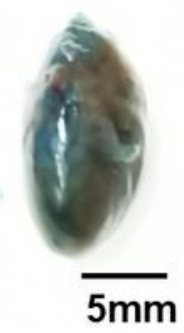

Figure 2

RvD1 pretreatment decreased myocardial injury induced by I/R in diabetic mice. A-B. RvD1 treatment has no influence on blood glucose and body weight. C. Representative TTC staining and Evans blue images of the hearts. D. Quantification of infarct size from different groups. E. Amount of Evans blue dye extracted from hearts from mice with $D M+I R$, and DM + IR + RvD1 treatment. F. Representative images 
for vascular permeability (Evans blue dye leakage) after IR and RvD1 treatment. $(n=6),{ }^{\star} p<0.05,{ }^{* \star} p<$ $0.01, * \star \star p<0.001$.
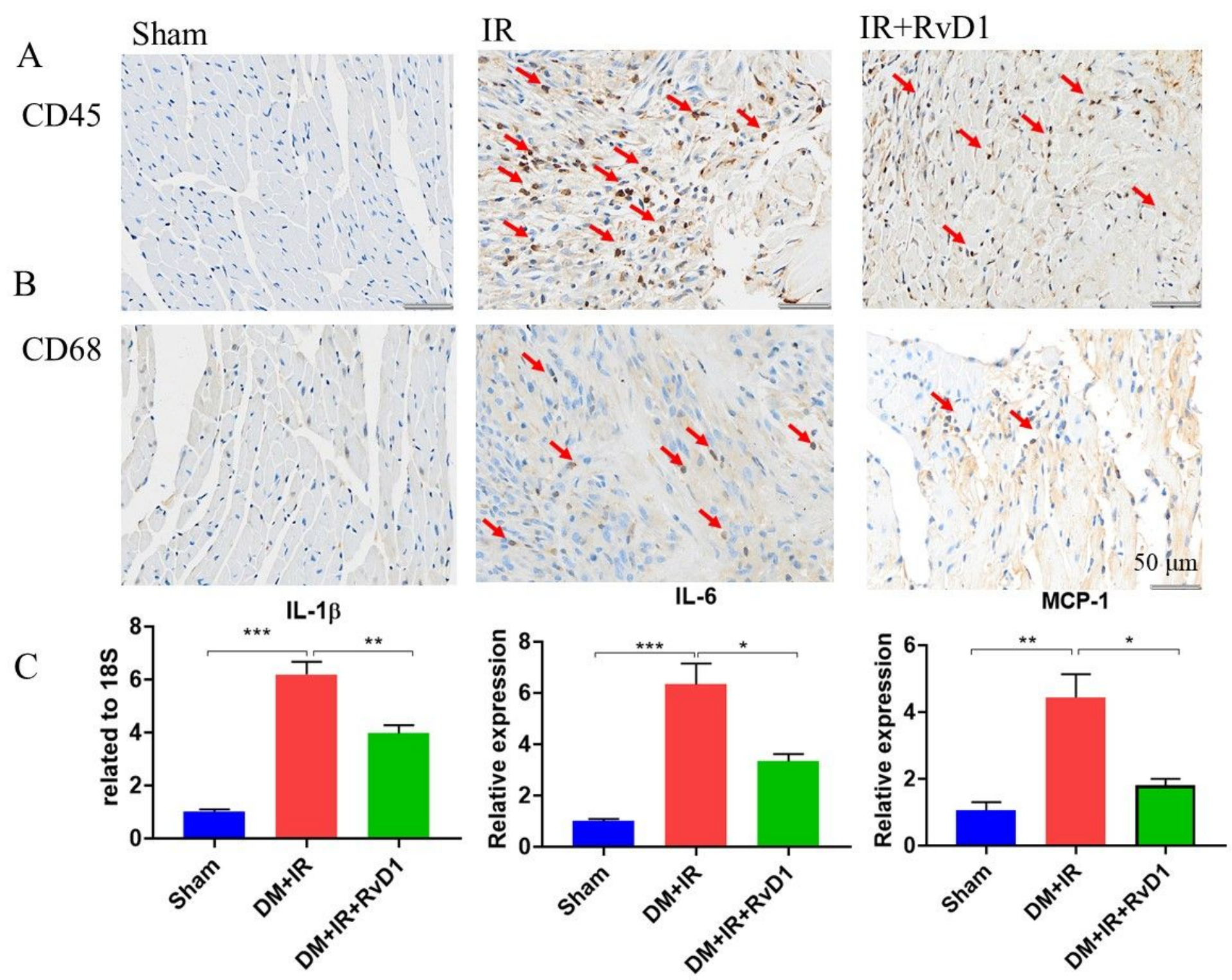

\section{Figure 3}

Effect of RvD1 pretreatment on inflammatory response in the heart subjected to I/R in diabetic mice. A. Representative histological images showing CD $45+$ leukocytes (the red arrows represent CD 45 positive cell and blue DAPI staining indicates nuclei) in the heart of each group. B. Representative histological images showing CD68 positive macrophages in the heart of each group. C. Changes in gene expression of inflammatory cytokines in the ischemic myocardium by quantitative real-time PCR, IL-1ß, IL-6, MCP-1. $(n=6),{ }^{*} p<0.05, * \star p<0.01, * \star \star p<0.001$. 


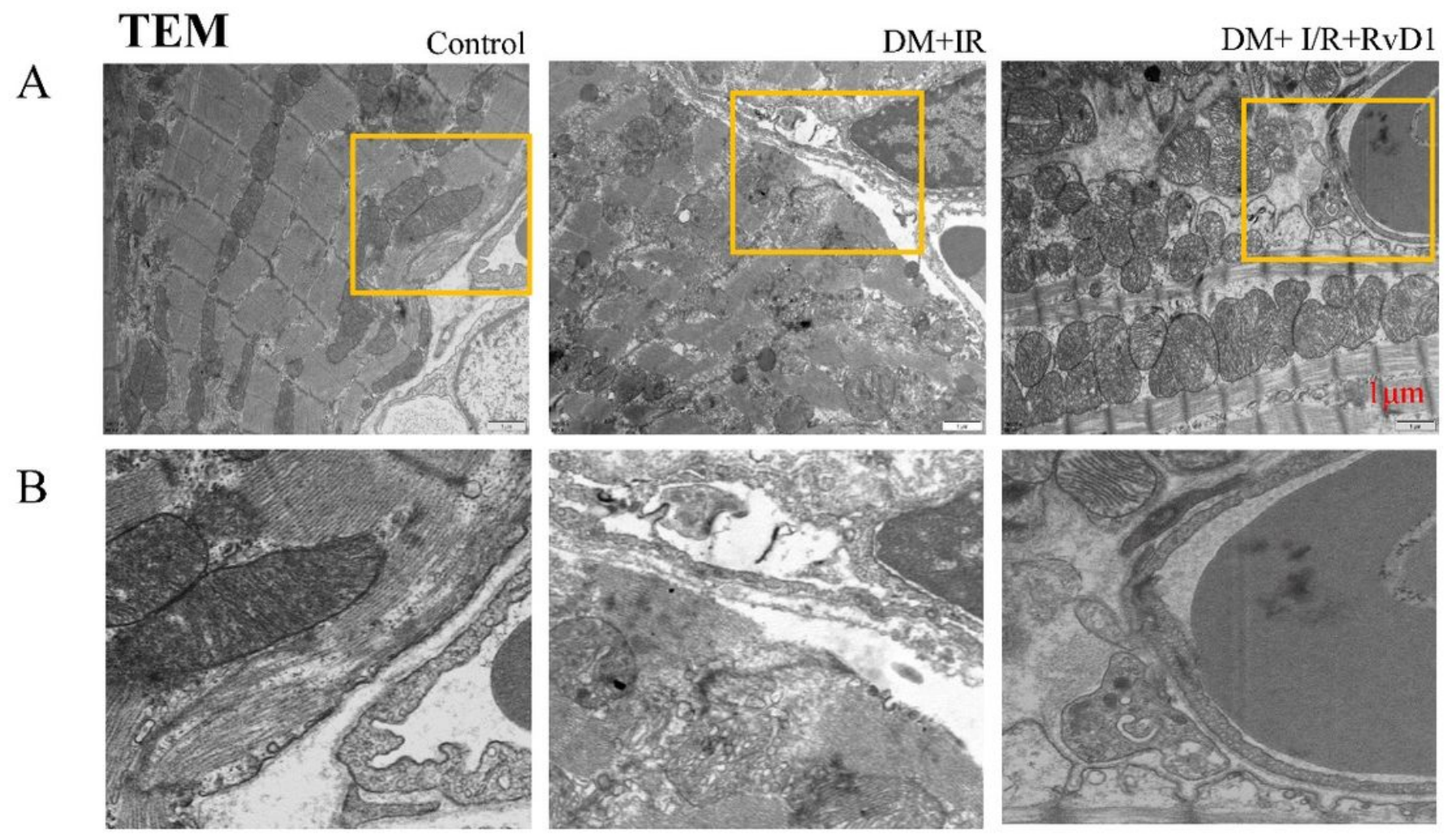

\section{Figure 4}

RvD1 pretreatment reduced I/R-mediated microvascular Damage in diabetes. A-B. TEM of microvascular structures after cardiac I/R injury, the yellow arrows indicate the damaged vascular wall. B. 
A
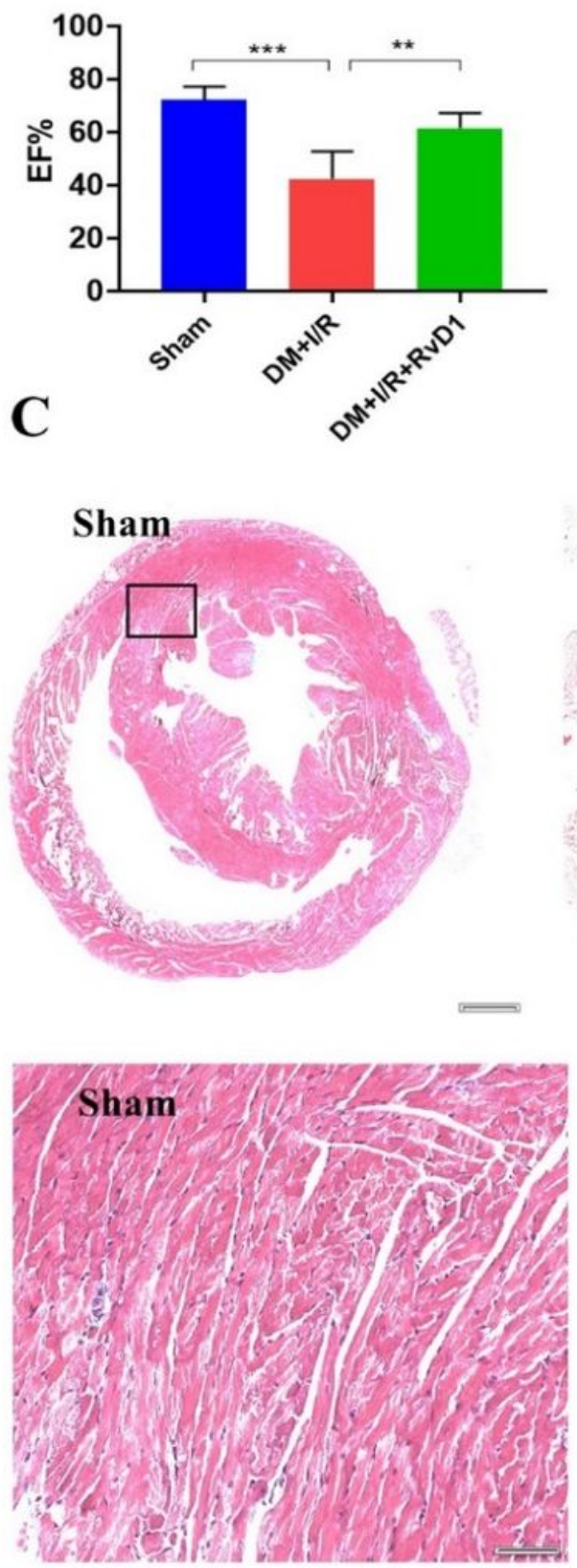

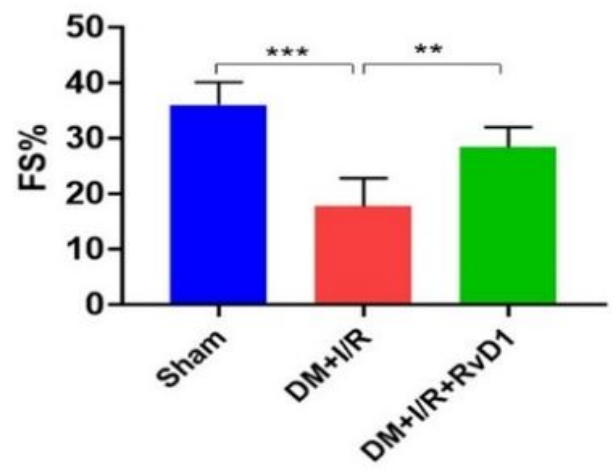

$\mathbf{D M}+\mathbf{I} / \mathbf{R}$
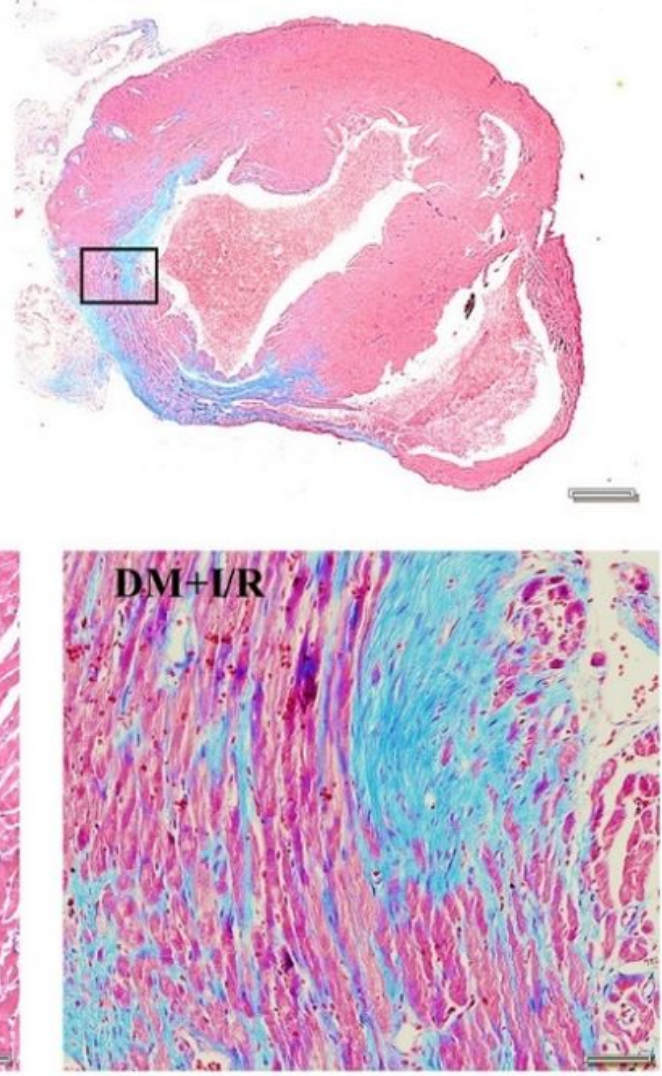

B

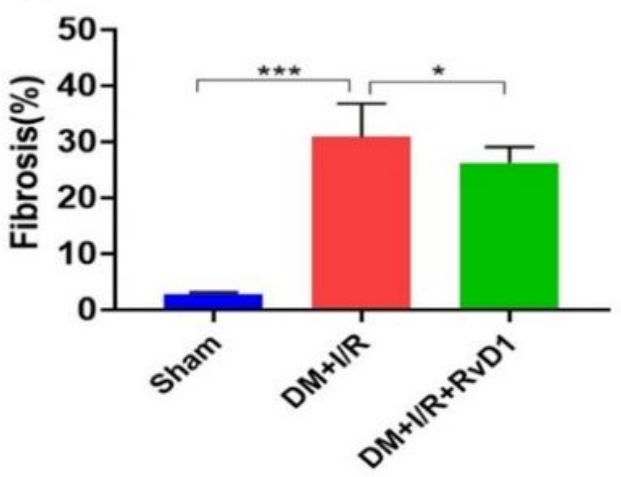

$\mathrm{DM}+\mathrm{I} / \mathrm{R}+\mathrm{RvD1}$
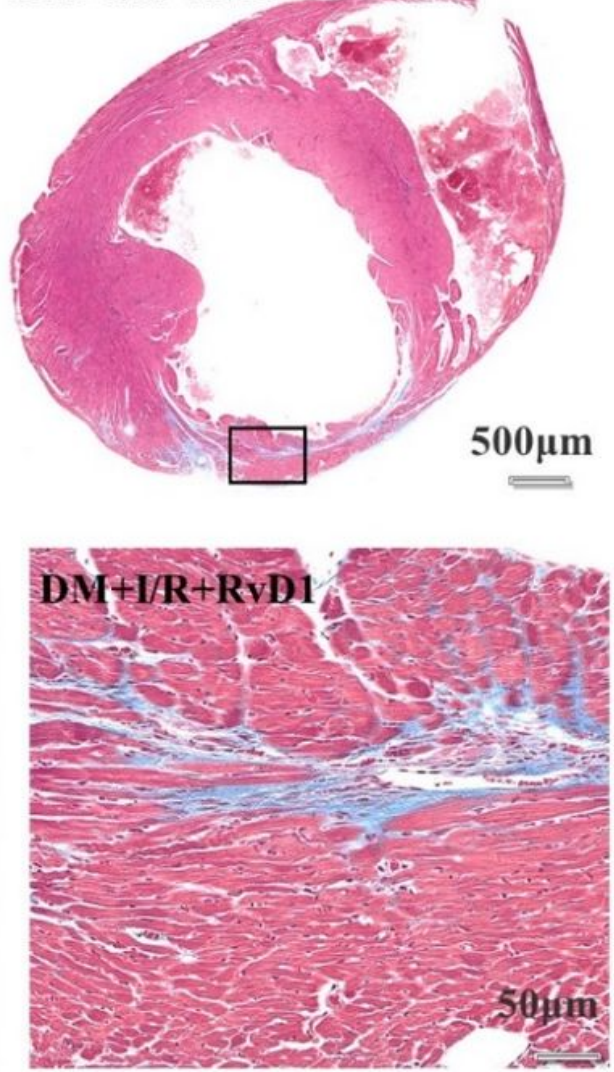

\section{Figure 5}

RvD1 treatment improve cardiac performance and alleviated chronic cardiac remodeling after IR in diabetic mice. A. Echocardiographic analysis at 2 weeks after RvD1 treatment in diabetic IR, EF, left ventricular ejection fraction; FS, left ventricular fractional shortening. B. Quantification of cardiac fibrosis from different groups. C. Masson staining for cardiac fibrosis after I/R. $(n=6),{ }^{\star} p<0.05,{ }^{\star \star} p<0.01,{ }^{\star \star \star} p<$ 0.001 . 
A
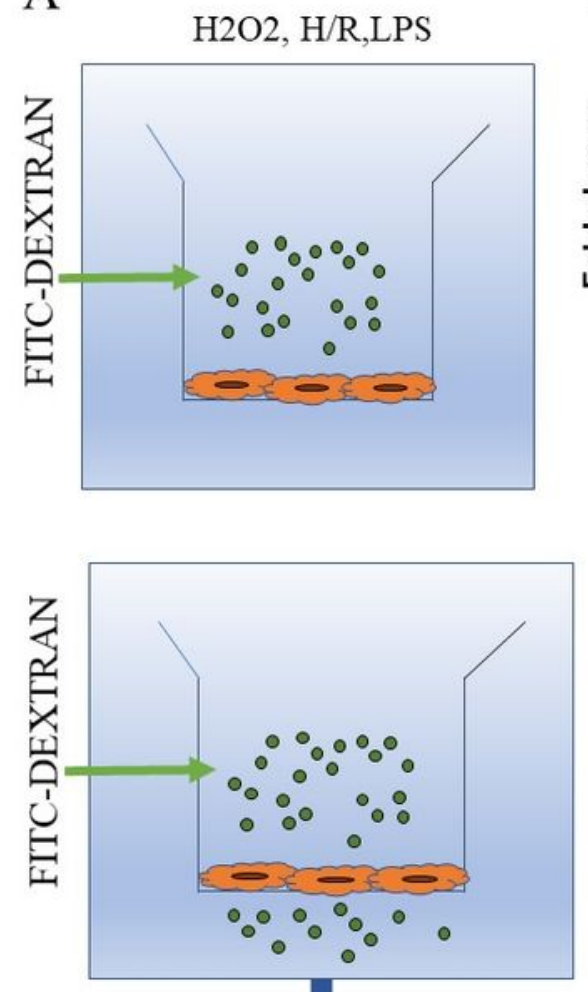

Collection for assay

$\mathrm{E}$

MCP-1

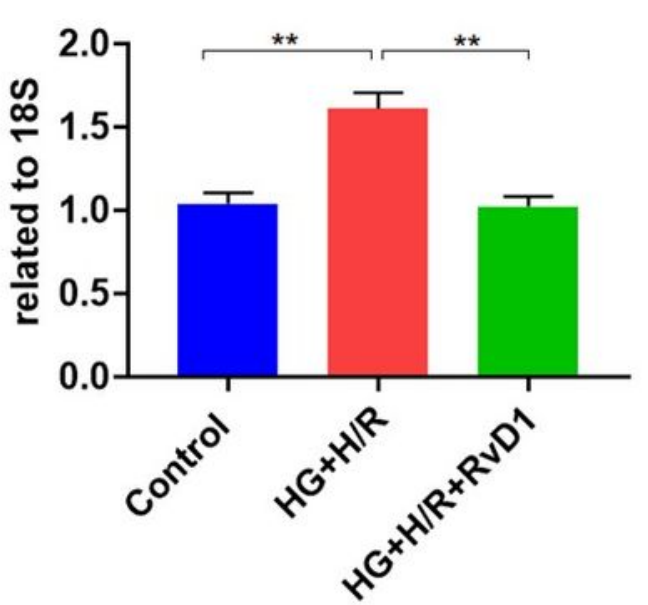

$\mathrm{B}$
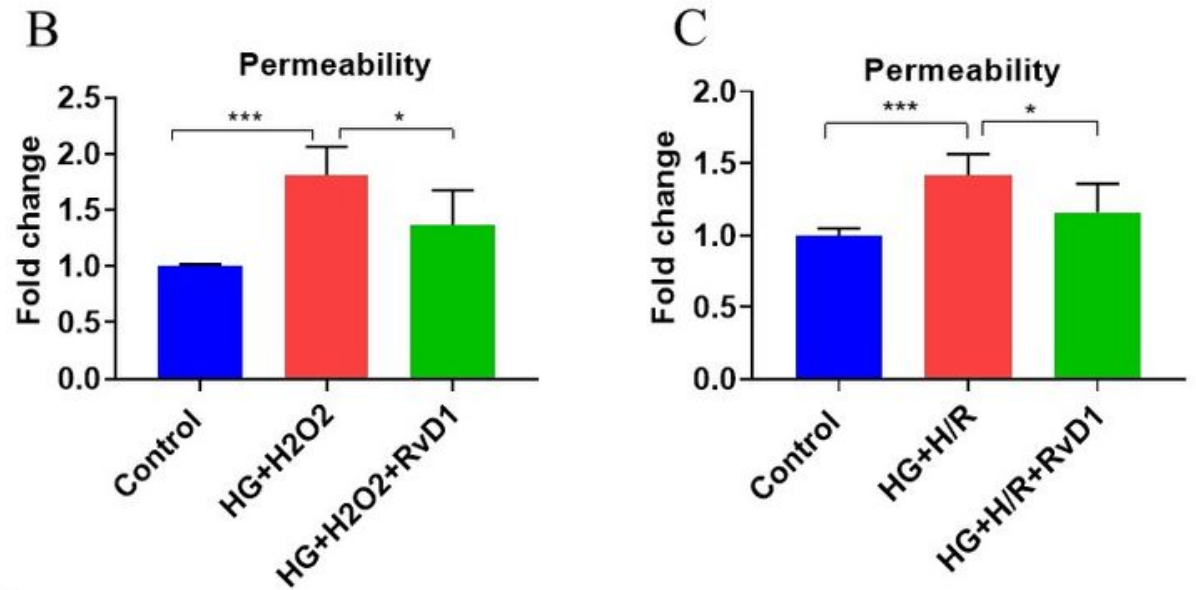

D

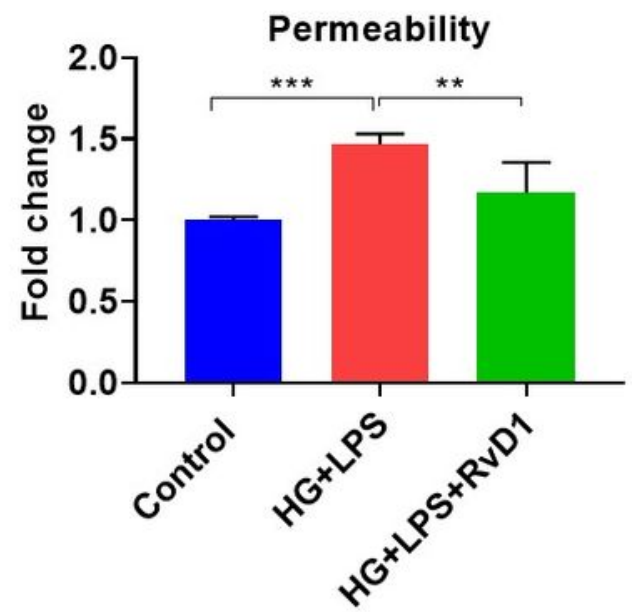

IL-1 $\beta$
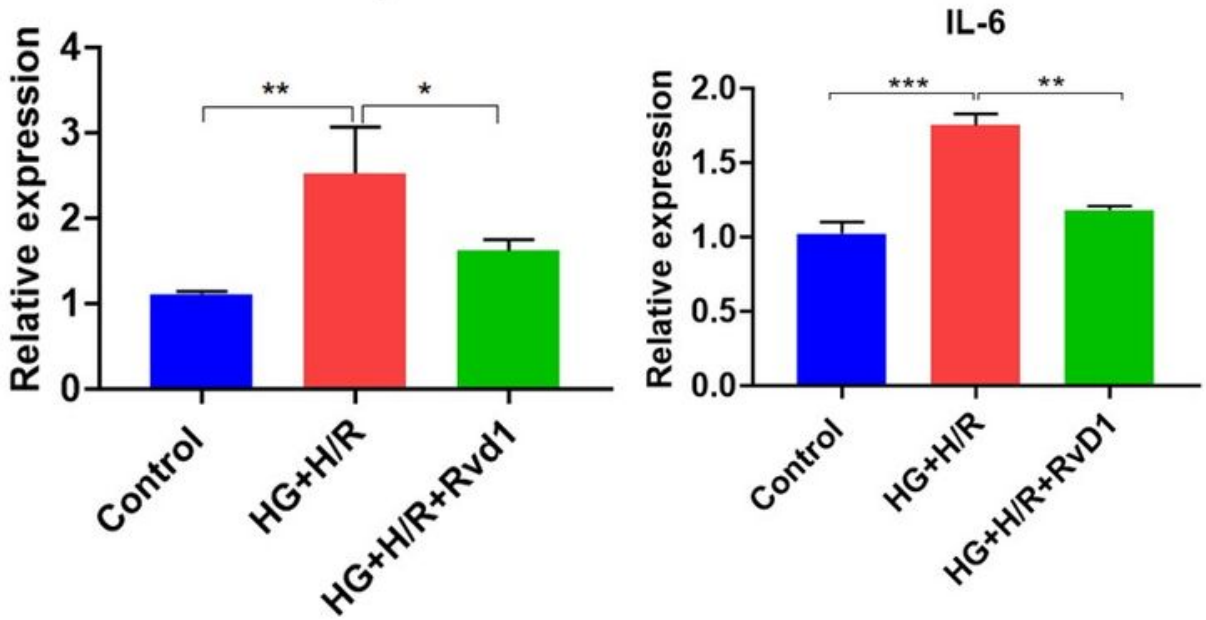

Figure 6

RvD1 reduced endothelial cell permeability stimulated with H2O2, H/R, and LPS. A. Transwell system used for the permeability experiments. B. Permeability of endothelial monolayer stimulated with hydrogen peroxide $(\mathrm{H} 2 \mathrm{O} 2,400 \mu \mathrm{M})$. C. hypoxia for 12 hours and re-oxygenation for 12 hours. D. LPS $(400 \mathrm{ng} / \mathrm{ml})$ for 12 hours, changes in the permeability was detected by measuring the fluorescence of FITC-dextran. $\mathrm{E}$. 
Q-PCR analysis the mRNA expression of H/R induced inflammatory marker genes MCP-1, IL-6 and IL-1 $\beta$ in high glucose condition. $(n=6),{ }^{*} p<0.05,{ }^{* *} p<0.01,{ }^{* *} p<0.001$.

A

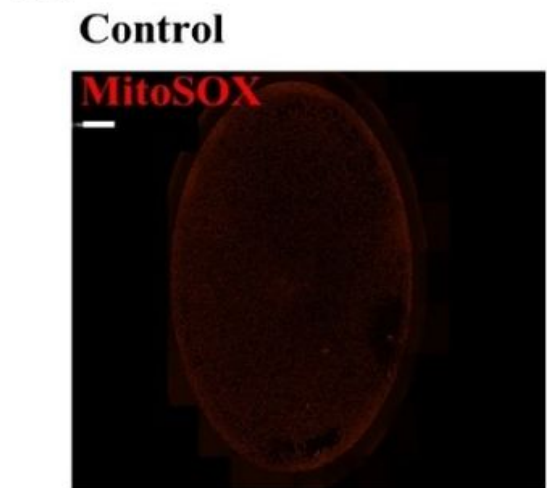

\section{$\mathrm{HG}+\mathrm{H} 2 \mathrm{O} 2$}

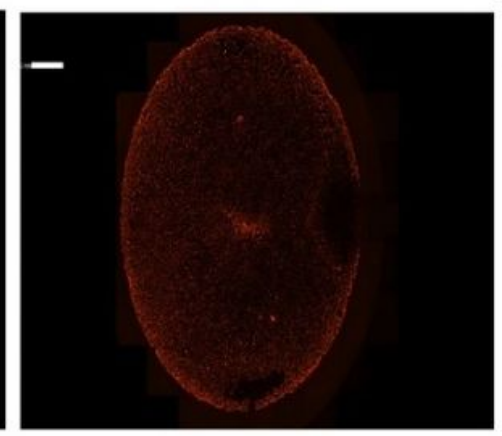

B

\section{Control}

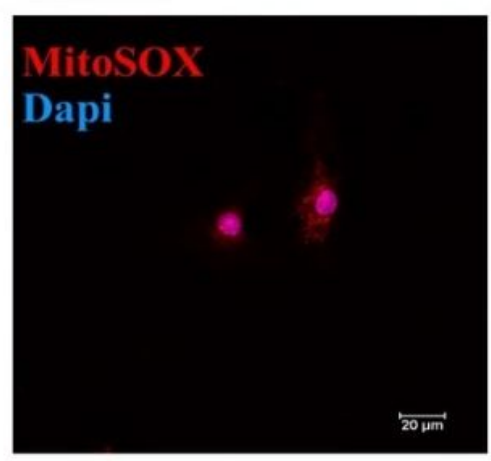

\section{$\mathrm{HG}+\mathrm{H} 2 \mathrm{O} 2$}

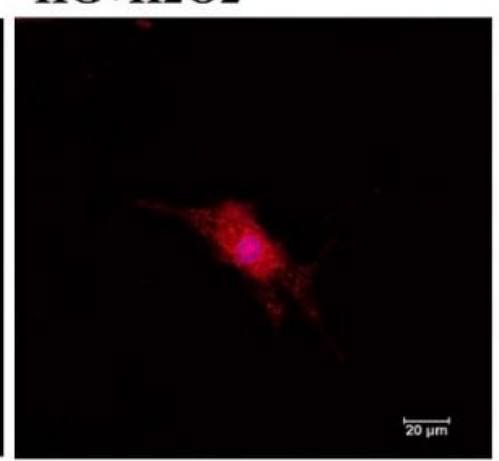

H2O2+HG+RvD1
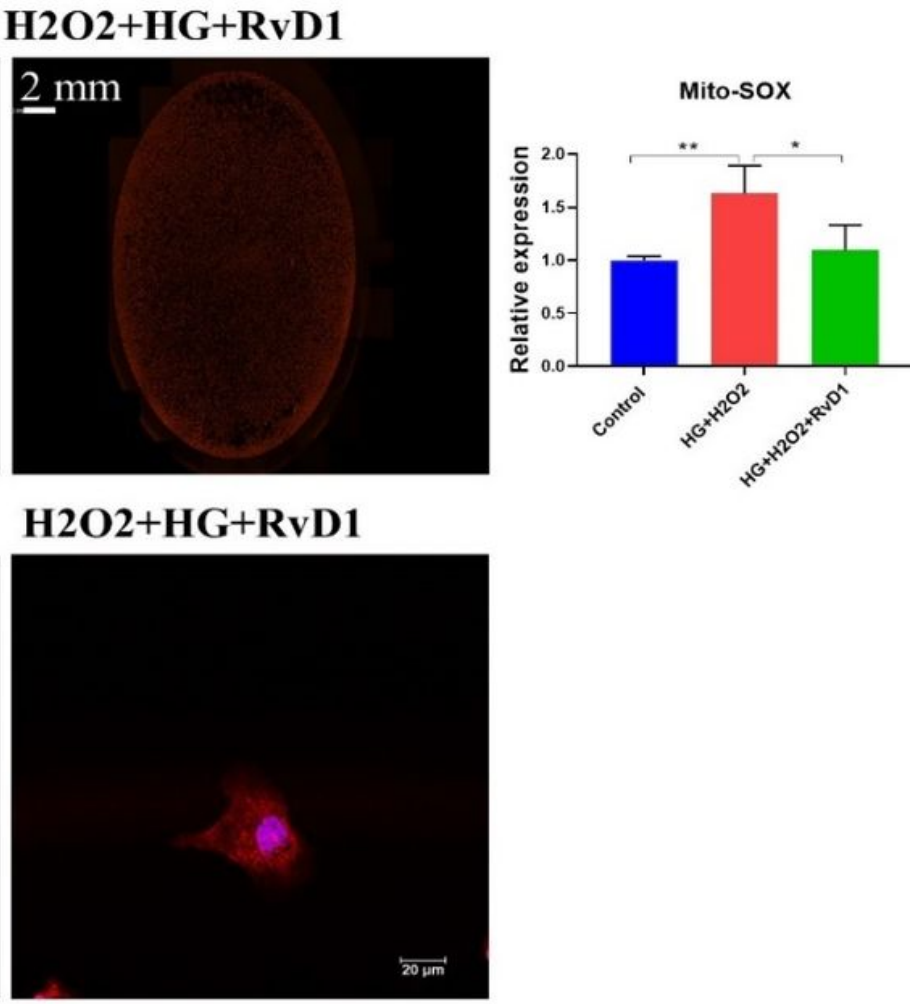

C

\section{Control}

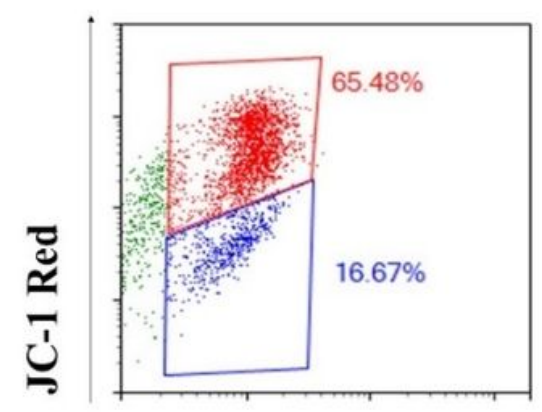

HG+H2O2

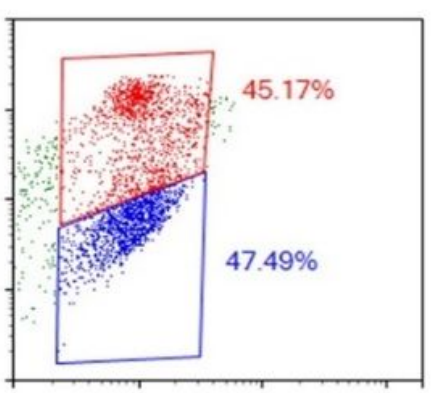

H2O2+HG+RvD1

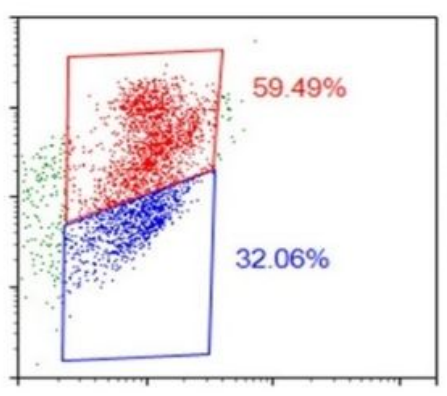

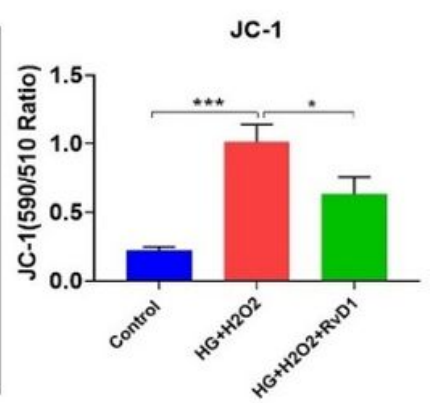

JC-1 Green

Figure 7

RvD1-mediated microvascular protection was regulated by mitochondrial damage. A. RvD1 decreased $\mathrm{H} 2 \mathrm{O} 2$-induced mitochondria reactive oxygen species (mito-ROS) in HCMECs with high glucose condition, mito-ROS determined by MitoSOX Red staining and Mito-SOX relative expression analysis by Celigo imaging cytometer $(n=4)$. B. MitoSOX Red staining images for each group. C. JC-1 analysis of mitochondrial transmembrane potential in HCMECs as determined by flow cytometry analysis, and JC-1 analysis was used green/red fluorescence ratio $(n=3)$. ${ }^{\star} p<0.05,{ }^{* \star} p<0.01,{ }^{\star \star *} p<0.001$. 


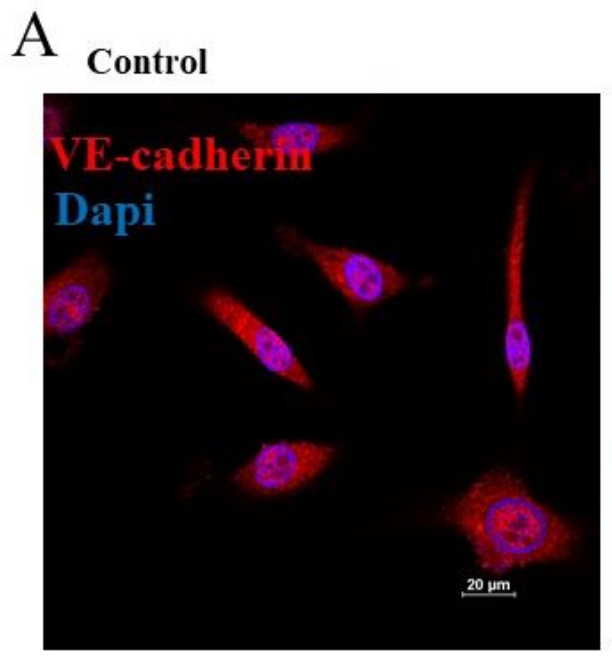

HG $+\mathbf{H} / \mathbf{R}$

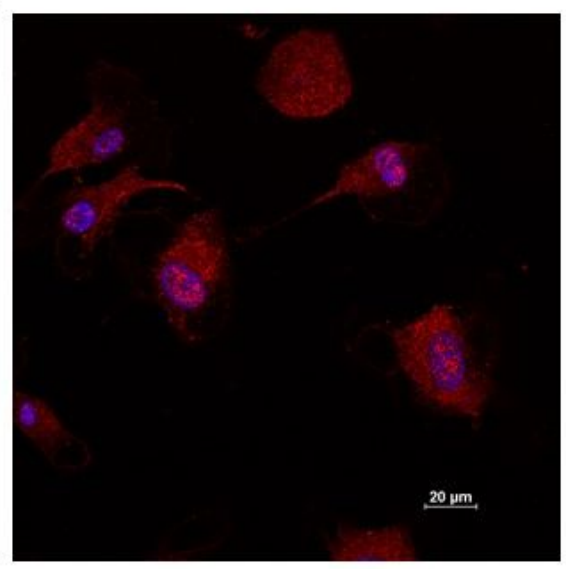

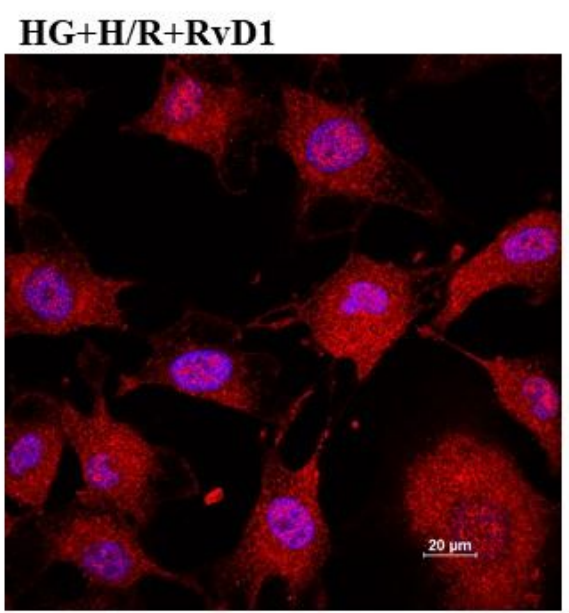

B
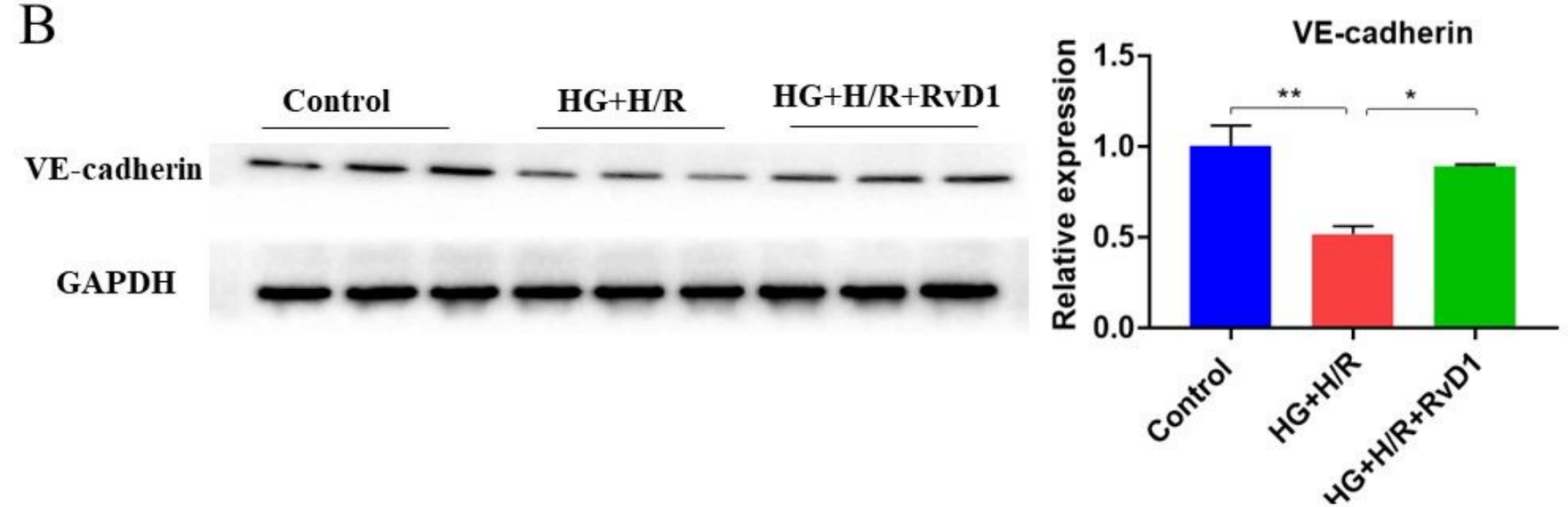

$\mathrm{C}$

VE-cadherin

\section{GAPDH}

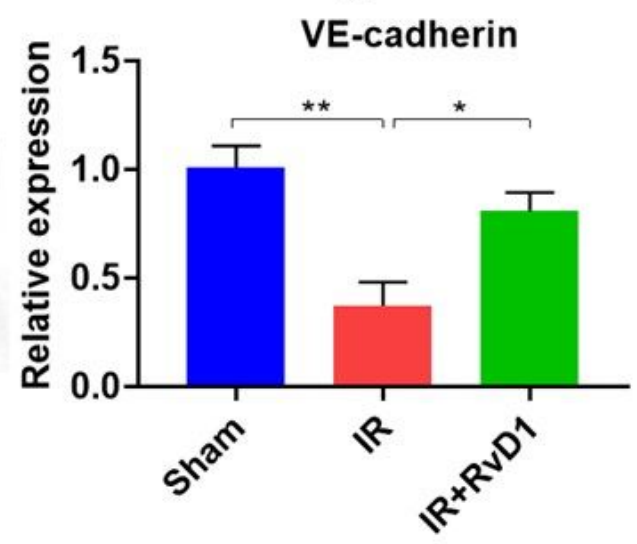

\section{Figure 8}

RvD1 decreased endothelial permeability with preservation of VE-cadherin. A. VE-cadherin immunostaining in H/R-induced HCMECs with or without RvD1 in high glucose condition. B. Western blots showed VE-cadherin in high glucose cultured HCMECs, 12 hypoxia and 12 re-oxygenation reduced VE-cadherin, which was reversed by RvD1. C. Western blots showed VE-cadherin expression in diabetic 
I/R hearts. RvD1 treatment increased VE-cadherin expression, and quantification data on myocardial VEcadherin expression. $(n=3),{ }^{*} p<0.05,{ }^{* *} p<0.01,{ }^{* \star} p<0.001$. 